\title{
Structure-based design of antiviral drug candidates targeting the SARS-CoV-2 main protease
}

\author{
Wenhao Dai',2*, Bing Zhang ${ }^{3 *}$, Xia-Ming Jiang ${ }^{4 *}$, Haixia Su', Jian Li',5, Yao Zhao ${ }^{3}$, Xiong Xie ${ }^{1}$, \\ Zhenming Jin ${ }^{3}$, Jingjing Peng ${ }^{1}$, Fengjiang Liu ${ }^{3}$, Chunpu Li', You Li ${ }^{6}$, Fang Bai ${ }^{3}$, Haofeng Wang ${ }^{3}$, \\ Xi Cheng', Xiaobo Cen ${ }^{6}$, Shulei Hu${ }^{1}$, Xiuna Yang ${ }^{3}$, Jiang Wang', Xiang Liu ${ }^{7}$, Gengfu Xiao ${ }^{4}$, \\ Hualiang Jiang, ${ }^{1,2,3}$, Zihe Rao ${ }^{3}$, Lei-Ke Zhang ${ }^{4} \uparrow$, Yechun Xu' ${ }^{1}$, Haitao Yang ${ }^{3} \uparrow$, Hong Liu ${ }^{1,2,5} \uparrow$
}

${ }^{1}$ State Key Laboratory of Drug Research, CAS Key Laboratory of Receptor Research, Shanghai Institute of Materia Medica, Chinese Academy of Sciences, Shanghai 201203, China. ${ }^{2}$ School of Pharmacy, China Pharmaceutical University, Nanjing 210009, Jiangsu, China. ${ }^{3}$ Shanghai Institute for Advanced Immunochemical Studies and School of Life Science and Technology, ShanghaiTech University, Shanghai 201210, China. ${ }^{4}$ State Key Laboratory of Virology, Wuhan Institute of Virology, Center for Biosafety Mega-Science, Chinese Academy of Sciences, Wuhan 430071, China. ${ }^{5}$ College of Pharmacy, Nanjing University of Chinese Medicine, Qixia District, Nanjing 210023, China. ${ }^{6}$ National Chengdu Center for Safety Evaluation of Drugs, Westchina Hospital of Sichuan Unverisity, High-Tech Development Zone, Chengdu, Sichuan 610041, China. ${ }^{7}$ State Key Laboratory of Medicinal Chemical Biology, Frontiers Science Center for Cell Response, College of Life Sciences, College of Pharmacy, Nankai University, Tianjin 300353, China.

*These authors contributed equally to this work.

†Corresponding author. Email: hliu@simm.ac.cn (H.L.); yanght@shanghaitech.edu.cn (H.Y.); zhangleike@wh.iov.cn (L.-K.Z.); ycxu@simm.ac.cn (Y.X.)

SARS-CoV-2 is the etiological agent responsible for the global COVID-19 outbreak. The main protease $\left(M^{\text {pro }}\right)$ of SARS-CoV-2 is a key enzyme that plays a pivotal role in mediating viral replication and transcription. We designed and synthesized two lead compounds (11a and 11b) targeting $\mathrm{M}^{\text {pro }}$. Both exhibited excellent inhibitory activity and potent anti-SARS-CoV-2 infection activity. The X-ray crystal structures of SARS-CoV-2 $\mathrm{M}^{\text {pro }}$ in complex with $11 \mathrm{a}$ or $11 \mathrm{~b}$, both determined at $1.5 \AA$ resolution, showed that the aldehyde groups of $11 \mathrm{a}$ and $11 \mathrm{~b}$ are covalently bound to Cys 145 of $\mathrm{M}^{\text {pro. }}$. Both compounds showed good PK properties in vivo, and 1la also exhibited low toxicity, suggesting that these compounds are promising drug candidates.

In late December 2019, a cluster of pneumonia cases caused by a novel coronavirus (CoV) was reported in Wuhan, China (1-3). Genomic sequencing showed that this pathogenic coronavirus is $96.2 \%$ identical to a bat coronavirus and shares $79.5 \%$ sequence identify to SARS-CoV (4-6). This novel coronavirus was named severe acute respiratory syndrome coronavirus 2 (SARS-CoV-2) by the International Committee on Taxonomy of Viruses, and the pneumonia was designated as COVID-19 by the World Health Organization (WHO) on February 11, 2020 (7). The epidemic spread rapidly to more than 212 countries and was announced as a global health emergency by WHO (8). No clinically effective vaccines or specific antiviral drugs are currently available for the prevention and treatment of COVID-19 infections. The combination of $\alpha$-interferon and the anti-HIV drugs Lopinavir/Ritonavir (Kaletra ${ }^{\circledR}$ ) has been used, but the curative effect remains very limited and there can be toxic side effects (9). Remdesivir, a broad-spectrum antiviral drug developed by Gilead Sciences, Inc., is also being explored for the treatment of COVID-19, but more data are needed to prove its efficacy (10-12). Specific anti-SARS-CoV-2 drugs with efficiency and safety are urgently needed.

A maximum likelihood tree based on the genomic sequence showed that the virus falls within the subgenus Sarbecovirus of the genus Betacoronavirus (6). Coronaviruses are enveloped, positive-sense, single-stranded RNA viruses. The genomic RNA of CoVs is approximately $30 \mathrm{k} \mathrm{nt}$ in length with a 5 '-cap structure and 3 ' -poly-A tail, and contains at least 6 open reading frames (ORFs) $(13,14)$. The first ORF (ORF 1a/b), about two-third of genome length, directly translates two polyproteins: ppla and pplab, because there is an a-1 frameshift between ORFla and ORF1b. These polyproteins are processed by a main protease $\left(\mathrm{M}^{\mathrm{pro}}\right)$, also known as the $3 \mathrm{C}$-like protease $\left(3 \mathrm{CL}^{\text {pro }}\right)$, and one or two papain-like proteases (PLPs), into 16 non-structural proteins (nsps). These nsps engage in the production of subgenomic RNAs that encode four main structural proteins (envelope (E), membrane (M), spike (S), and nucleocapsid (N) pro- 
teins) and other accessory proteins $(15,16)$. Therefore, these proteases, especially $\mathrm{M}^{\text {pro }}$, play a vital role in the life cycle of coronavirus.

$\mathrm{M}^{\text {pro }}$ is a three-domain (domains I to III) cysteine protease involved in most maturation cleavage events within the precursor polyprotein (17-19). Active $\mathrm{M}^{\text {pro }}$ is a homodimer containing two protomers. The $\mathrm{CoV} \mathrm{M}^{\text {pro }}$ features a noncanonical Cys-His dyad located in the cleft between domains I and II (17-19). $\mathrm{M}^{\text {pro }}$ is conserved among CoVs and several common features are shared among the substrates of $\mathrm{M}^{\text {pro }}$ in different CoVs. The amino acids in substrates from the $\mathrm{N}$ terminus to $\mathrm{C}$ terminus are numbered as fellows (-P4-P3-P2$\left.\mathrm{P} 1 \downarrow \mathrm{P} 1^{\prime}-\mathrm{P} 2{ }^{\prime}-\mathrm{P} 3^{\prime}-\right)$, and the cleavage site is between the P1 and $\mathrm{P}^{\prime}$. In particular, a Gln residue is almost always required in the P1 position of the substrates. There is no human homolog of $\mathrm{M}^{\text {pro }}$ which makes it an ideal antiviral target (20-22).

The active sites of $\mathrm{M}^{\text {pro }}$ are highly conserved among all CoV's $\mathrm{M}^{\text {pro }} \mathrm{S}$ and are usually composed of four sites ( $\mathrm{S} 1^{\prime}, \mathrm{S} 1$, S2 and S4) (22). By analyzing the substrate-binding pocket of SARS-CoV M ${ }^{\text {pro }}$ (PDB ID: 2H2Z), novel inhibitors targeting the SARS-CoV-2 $\mathrm{M}^{\text {pro }}$ were designed and synthesized (Fig. 1). The thiol of a cysteine residue in the $\mathrm{S}^{\prime}$ ' sites anchors inhibitors by a covalent linkage that is important for the inhibitors to maintain antiviral activity. In our design of new inhibitors, an aldehyde was selected as a new warhead in P1 in order to form a covalent bond with cysteine. The reported SARS-CoV $\mathrm{M}^{\text {pro }}$ inhibitors often have an $(S)-\gamma-$ lactam ring that occupies the S1 site of $\mathrm{M}^{\text {pro }}$, and this ring was expected to be a good choice in P1 (23). Furthermore, the S2 site of coronavirus $\mathrm{M}^{\mathrm{pro}}$ is usually large enough to accommodate the bigger P2 fragment. To test the importance of different ring systems, a cyclohexyl or 3fluorophenyl were introduced in P2, with the fluorine expected to enhance activity. An indole group was introduced into P3 in order to form new hydrogen bonds with S4 and improve drug-like properties.

The synthetic route and chemical structures of the compounds (11a and 11b) are shown in scheme S1. The starting material ( $N$-Boc- $L$-glutamic acid dimethyl ester 1) was obtained from commercial suppliers and used without further purification to synthesize the key intermediate $\mathbf{3}$ according to the literature (24). The intermediates $\mathbf{6 a}$ and $\mathbf{6 b}$ were synthesized from $\mathbf{4}$ and acids $5 \mathbf{a}, \mathbf{5 b}$. Removal of the $t$-butoxycarbonyl group from $\mathbf{6 a}$ and $\mathbf{6 b}$ yielded $7 \mathbf{a}$ and $\mathbf{7 b}$. Coupling $\mathbf{7 a}$ and $\mathbf{7 b}$ with the acid $\mathbf{8}$ yielded the esters $\mathbf{9 a}$ and $\mathbf{9 b}$. The peptidomimetic aldehydes $\mathbf{1 1 a}$ and $\mathbf{1 1 b}$ were approached through a two-step route in which the ester derivatives 9 were first reduced with $\mathrm{NaBH}_{4}$ to generate the primary alcohols $\mathbf{1 0 a}$ and 10b, which were subsequently oxidized into aldehydes $\mathbf{1 1 a}$ and $\mathbf{1 1 b}$ with Dess-Martin Periodinane (DMP).
Recombinant SARS-CoV-2 $\mathrm{M}^{\text {pro }}$ was expressed and purified from Escherichia coli (E. coli) (18, 25). A fluorescently labeled substrate, MCA-AVLQ $\downarrow$ SGFR-Lys (Dnp)-Lys- $\mathrm{NH}_{2}$, derived from the $N$-terminal auto-cleavage sequence from the viral protease was designed and synthesized for the enzymatic assay.

Both $11 a$ and $11 b$ exhibited high SARS-CoV-2 $\mathrm{M}^{\text {pro }}$ inhibition activity, which reached $100 \%$ for $\mathbf{1 1 a}$ and $96 \%$ for $\mathbf{1 1 b}$ at $1 \mu \mathrm{M}$, respectively. We used a fluorescence resonance energy transfer (FRET)-based cleavage assay to determine the $\mathrm{IC}_{50}$ values. The results revealed excellent inhibitory potency with $\mathrm{IC}_{50}$ values of $0.053 \pm 0.005 \mu \mathrm{M}$ and $0.040 \pm 0.002 \mu \mathrm{M}$, for $\mathbf{1 1 a}$ and $\mathbf{1 1 b}$ respectively (Fig. 2).

In order to elucidate the mechanism of inhibition of SARS-CoV-2 $\mathrm{M}^{\text {pro }}$ by 11a, we determined the high-resolution crystal structure of this complex at $1.5-\AA$ resolution (table S1). The crystal of $\mathrm{M}^{\text {pro }}-\mathbf{1 1 a}$ belong to the space group $C 2$ and an asymmetric unit contains only one molecule (table S1). Two molecules (designated protomer $\mathrm{A}$ and protomer $\mathrm{B}$ ) associate into a homodimer around a crystallographic 2-fold symmetry axis (fig. S2). The structure of each protomer contains three domains with the substrate-binding site located in the cleft between domain I and II. At the active site of SARS-CoV-2 $\mathrm{M}^{\text {pro }}$, Cys145 and His41 (Cys-His) form a catalytic dyad (fig. S2).

The electron density map clearly showed compound $\mathbf{1 1 a}$ in the substrate binding pocket of SARS-CoV-2 $\mathrm{M}^{\text {pro }}$ in an extended conformation (Fig. 3A and fig. S3, A and B). Details of the interaction are shown in Fig. 3, B and C. The electron density shows that the $\mathrm{C}$ of the aldehyde group of $11 a$ and the catalytic site Cys145 of SARS-CoV-2 $\mathrm{M}^{\text {pro }}$ form a standard 1.8-A C-S covalent bond. The oxygen atom of the aldehyde group also plays a crucial role in stabilizing the conformations of the inhibitor by forming a 2.9 -Å hydrogen bond with the backbone of residues Cys145 in the $\mathrm{S} 1$ ' site. The $(S)$ - $\gamma$-lactam ring of $\mathbf{1 1 a}$ at P1 fits well into the S1 site. The oxygen of the $(S)$ - $\gamma$-lactam group forms a $2.7-\AA \AA$ hydrogen bond with the side chain of His163. The main chain of Phe140 and side chain of Glu166 also participate in stabilizing the $(S)$ - $\gamma$-lactam ring by forming $3.2-\AA$ and $3.0-\AA$ hydrogen bonds with its $\mathrm{NH}$ group, respectively. In addition, the amide bonds on the chain of $\mathbf{1 1 a}$ are hydrogen-bonded with the main chains of His164 (3.2 $\AA)$ and Glu166 (2.8 $\AA$ ), respectively. The cyclohexyl moiety of 11 at P2 deeply inserts into the S2 site, stacking with the imidazole ring of His41. The cyclohexyl group is also surrounded by the side chains of Met49, Tyr54, Met165, Asp187 and Arg188, producing extensive hydrophobic interactions. The indole group of $\mathbf{1 1 a}$ at P3 is exposed to solvent (S4 site) and is stabilized by Glu166 through a 2.6- $\AA$ hydrogen bond. The side chains of residues Pro168 and Gln189 interact with the indole group of 11a through hydrophobic interactions. Interestingly, multiple 
water molecules (named W1-W6) play an important role in binding 11a. W1 interacts with the amide bonds of 11a through a $2.9-\AA \AA$ hydrogen bond, whereas W2-6 form a number of hydrogen bonds with the aldehyde group of $\mathbf{1 1 a}$ and the residues of Asn142, Gly143, Thr26, Thr25, His41 and Cys44, which contributes to stabilizing $\mathbf{1 1 a}$ in the binding pocket.

The crystal structure of SARS-CoV-2 $\mathrm{M}^{\text {pro }}$ in complex with $\mathbf{1 1 b}$ is very similar to that of the 11a complex and shows a similar inhibitor binding mode (Fig. 3D and figs. S3, C and D, and S4A). The difference in binding mode is most probably due to the 3-fluorophenyl group of $\mathbf{1 1 b}$ at $\mathrm{P} 2$. Compared with the cyclohexyl group in 11a, the 3fluorophenyl group undergoes a significant downward rotation (Fig. 3D). The side chains of residues His41, Met49, Met165, Val186, Asp187 and Arg188 interact with this aryl group through hydrophobic interactions and the side chain of Gln189 stabilizes the 3-fluorophenyl group with an additional 3.0-Å hydrogen bond (Fig. 3, E and F). In short, these two crystal structures reveal a similar inhibitory mechanism in which both compounds occupy the substrate-binding pocket and block the enzyme activity of SARS-CoV-2 $\mathrm{M}^{\text {pro }}$.

Compared with those of N1, N3 and N9 in SARS-CoV $\mathrm{M}^{\text {pro }}$ complex structures reported previously, the binding modes of $\mathbf{1 1 a}$ and $\mathbf{1 1 b}$ in SARS-CoV-2 $\mathrm{M}^{\text {pro }}$ complex structures are similar and the differences among these overall structures are small (Fig. 4 and fig. S4, B to F) (22). The differences mainly lie in the interactions at $\mathrm{S} 1$ ', $\mathrm{S} 2$ and $\mathrm{S} 4$ subsites, possibly due to various sizes of functional groups at corresponding P1', P2 and P4 sites in the inhibitors (Fig. 4, $\mathrm{A}$ and $\mathrm{C}$ ).

To further substantiate the enzyme inhibition results, we evaluated the ability of these compounds to inhibit SARS-CoV-2 in vitro (Fig. 5 and fig. S5). As shown in Fig. 5, compounds 11 a and $11 b$ exhibited good anti-SARS-CoV-2infection activity in cell culture with $\mathrm{EC}_{50}$ values of $0.53 \pm$ $0.01 \mu \mathrm{M}$ and $0.72 \pm 0.09 \mu \mathrm{M}$ using plaque-reduction assay, respectively. Neither compound caused significant cytotoxicity, with half cytotoxic concentration $\left(\mathrm{CC}_{50}\right)$ values of $>100$ $\mu \mathrm{M}$, yielding selectivity indices (SI) for $\mathbf{1 1 a}$ and $\mathbf{1 1 b}$ of $>189$ and $>139$, respectively. Both immunofluorescence and quantitative real-time PCR were also employed to monitor the antiviral activity of $\mathbf{1 1 a}$ and $\mathbf{1 1 b}$. The results show $\mathbf{1 1 a}$ and 11b exhibit a good antiviral effect on SARS-CoV-2 (Fig. 5 and fig. S5).

To explore the further druggability of the compounds $11 a$ and 11b, both of the compounds were evaluated for their pharmacokinetic (PK) properties. As shown in table S2, compound 11 a given intraperitoneally $(5 \mathrm{mg} / \mathrm{kg}$ ) and intravenously $(5 \mathrm{mg} / \mathrm{kg})$ displayed a half-life $\left(\mathrm{T}_{1 / 2}\right)$ of 4.27 hours and 4.41 hours, respectively, and a high maximal concentration $\left(\mathrm{C}_{\max }=2394 \mathrm{ng} / \mathrm{mL}\right)$ and a good bioavailability of
$87.8 \%$ were observed when the compound $11 a$ was given intraperitoneally. Metabolic stability of $\mathbf{1 1 a}$ in mice was also good $($ Clearance $(\mathrm{CL})=17.4 \mathrm{~mL} / \mathrm{min} / \mathrm{mg})$. When administered intraperitoneally $(20 \mathrm{mg} / \mathrm{kg})$, subcutaneously (5 $\mathrm{mg} / \mathrm{kg}$ ) and intravenously ( $5 \mathrm{mg} / \mathrm{kg}$ ), compound $\mathbf{1 1 b}$ also showed good PK properties (the bioavailability of intraperitoneally and subcutaneously are more than $80 \%$, and a longer $\mathrm{T}_{1 / 2}$ of 5.21 hours when $\mathbf{1 1 b}$ was given intraperitoneally). Considering the danger of COVID-19, we selected the intravenous drip administration to further study for the reason that value of the area under the curve (AUC) is high and the effect is rapid. Compared with 11a administrated intravenously, the $T_{1 / 2}(1.65 \mathrm{~h})$ of $\mathbf{1 1 b}$ is shorter and the clearance rate is faster $(\mathrm{CL}=20.6 \mathrm{~mL} / \mathrm{min} / \mathrm{mg}$ ). Compound $11 a$ was selected for further investigation with intravenous drip dosing in Sprague-Dawley (SD) rats and Beagle dogs. The results showed (table $\mathrm{S} 3$ ) that $11 a$ exhibited long $\mathrm{T}_{1 / 2}$ (SD rat, 7.6 hours and Beagle dog, 5.5h), low clearance rate (rat, $4.01 \mathrm{~mL} / \mathrm{min} / \mathrm{kg}$ and $\mathrm{dog}, 5.8 \mathrm{~mL} / \mathrm{min} / \mathrm{kg}$ ) and high AUC value (rat, 41500 hours*ng/mL and dog, 14900 hours*ng/mL)). Those above PK results indicate that compound $\mathbf{1 1 a}$ is worth to warrant further study.

An in vivo toxicity study (table S4) of $\mathbf{1 1 a}$ has been carried out on SD rats and Beagle dogs. The acute toxicity of $11 a$ was measured on SD rats. No SD rats died after receiving $40 \mathrm{mg} / \mathrm{kg}$ by intravenous drip administration. When the dosage was raised to $60 \mathrm{mg} / \mathrm{kg}$, one of four SD rats died. The dose range toxicity study of $\mathbf{1 1 a}$ was conducted for seven days at dosing levels of 2,6 , and $18 \mathrm{mg} / \mathrm{kg}$ on SD rats and at $10-40 \mathrm{mg} / \mathrm{kg}$ on Beagle dogs. All animals received once daily dosing (QD), by intravenous drip, and all animals were clinically observed at least once a day. No obvious toxicity was observed in either group. These above data indicated that $11 a$ is good candidate for further clinical studies.

\section{REFERENCES AND NOTES}

1. N. Zhu, D. Zhang, W. Wang, X. Li, B. Yang, J. Song, X. Zhao, B. Huang, W. Shi, R. Lu, P. Niu, F. Zhan, X. Ma, D. Wang, W. Xu, G. Wu, G. F. Gao, W. Tan, A Novel Coronavirus from Patients with Pneumonia in China, 2019. N. Engl. J. Med. 382, 727-733 (2020). doi:10.1056/NEJMoa2001017 Medline

2. Q. Li, X. Guan, P. Wu, X. Wang, L. Zhou, Y. Tong, R. Ren, K. S. M. Leung, E. H. Y. Lau, J. Y. Wong, X. Xing, N. Xiang, Y. Wu, C. Li, Q. Chen, D. Li, T. Liu, J. Zhao, M. Liu, W. Tu, C. Chen, L. Jin, R. Yang, Q. Wang, S. Zhou, R. Wang, H. Liu, Y. Luo, Y. Liu, G. Shao, H. Li, Z. Tao, Y. Yang, Z. Deng, B. Liu, Z. Ma, Y. Zhang, G. Shi, T. T. Y. Lam, J. T. Wu, G. F. Gao, B. J. Cowling, B. Yang, G. M. Leung, Z. Feng, Early Transmission Dynamics in Wuhan, China, of Novel Coronavirus-Infected Pneumonia. N. Engl. J. Med. 382, 1199-1207 (2020). doi:10.1056/NEJMoa2001316 Medline

3. J. F. W. Chan, S. Yuan, K.-H. Kok, K. K.-W. To, H. Chu, J. Yang, F. Xing, J. Liu, C. C.Y. Yip, R. W.-S. Poon, H.-W. Tsoi, S. K.-F. Lo, K.-H. Chan, V. K.-M. Poon, W.-M. Chan, J. D. Ip, J.-P. Cai, V. C.-C. Cheng, H. Chen, C. K.-M. Hui, K.-Y. Yuen, A familial cluster of pneumonia associated with the 2019 novel coronavirus indicating person-to-person transmission: A study of a family cluster. Lancet 395, 514-523 (2020). doi:10.1016/S0140-6736(20)30154-9 Medline 
4. P. Zhou, X.-L. Yang, X.-G. Wang, B. Hu, L. Zhang, W. Zhang, H.-R. Si, Y. Zhu, B. Li, C.-L. Huang, H.-D. Chen, J. Chen, Y. Luo, H. Guo, R.-D. Jiang, M.-Q. Liu, Y. Chen, X.-R. Shen, X. Wang, X.-S. Zheng, K. Zhao, Q.-J. Chen, F. Deng, L.-L. Liu, B. Yan, F.-X. Zhan, Y.-Y. Wang, G.-F. Xiao, Z.-L. Shi, A pneumonia outbreak associated with a new coronavirus of probable bat origin. Nature 579, 270-273 (2020). doi:10.1038/s41586-020-2012-7 Medline

5. F. Wu, S. Zhao, B. Yu, Y.-M. Chen, W. Wang, Z.-G. Song, Y. Hu, Z.-W. Tao, J.-H. Tian, Y.-Y. Pei, M.-L. Yuan, Y.-L. Zhang, F.-H. Dai, Y. Liu, Q.-M. Wang, J.-J. Zheng, L. Xu, E. C. Holmes, Y.-Z. Zhang, A new coronavirus associated with human respiratory disease in China. Nature 579, 265-269 (2020). doi:10.1038/s41586-020-20083 Medline

6. R. Lu, X. Zhao, J. Li, P. Niu, B. Yang, H. Wu, W. Wang, H. Song, B. Huang, N. Zhu, Y. Bi, X. Ma, F. Zhan, L. Wang, T. Hu, H. Zhou, Z. Hu, W. Zhou, L. Zhao, J. Chen, Y. Meng, J. Wang, Y. Lin, J. Yuan, Z. Xie, J. Ma, W. J. Liu, D. Wang, W. Xu, E. C. Holmes, G. F. Gao, G. Wu, W. Chen, W. Shi, W. Tan, Genomic characterisation and epidemiology of 2019 novel coronavirus: Implications for virus origins and receptor binding. Lancet 395, 565-574 (2020). doi:10.1016/S01406736(20)30251-8 Medline

7. A. E. Gorbalenya, S. C. Baker, R. S. Baric, R. J. de Groot, C. Drosten, A. A. Gulyaeva, B. L. Haagmans, C. Lauber, A. M. Leontovich, B. W. Neuman, D. Penzar, S. Perlman, L. L. M. Poon, D. Samborskiy, I. A. Sidorov, I. Sola, J. Ziebuhr, Severe acute respiratory syndrome-related coronavirus: The species and its viruses-a statement of the Coronavirus Study Group. bioRxiv 2020.02.07.937862 [preprint]. 11 February 2020

8. World Health Organization, "WHO Director-General's opening remarks at the media briefing on COVID-19-11 March 2020" (2020);

www.who.int/dg/speeches/detail/who-director-general-s-opening-remarks-atthe-media-briefing-on-covid-19---11-march-2020.

9. B. Cao, Y. Wang, D. Wen, W. Liu, J. Wang, G. Fan, L. Ruan, B. Song, Y. Cai, M. Wei, X. Li, J. Xia, N. Chen, J. Xiang, T. Yu, T. Bai, X. Xie, L. Zhang, C. Li, Y. Yuan, H. Chen, H. Li, H. Huang, S. Tu, F. Gong, Y. Liu, Y. Wei, C. Dong, F. Zhou, X. Gu, J. Xu, Z. Liu, Y. Zhang, H. Li, L. Shang, K. Wang, K. Li, X. Zhou, X. Dong, Z. Qu, S. Lu, X. Hu, S. Ruan, S. Luo, J. Wu, L. Peng, F. Cheng, L. Pan, J. Zou, C. Jia, J. Wang, X. Liu, S. Wang, X. Wu, Q. Ge, J. He, H. Zhan, F. Qiu, L. Guo, C. Huang, T. Jaki, F. G. Hayden, P. W. Horby, D. Zhang, C. Wang, A Trial of Lopinavir-Ritonavir in Adults Hospitalized with Severe Covid-19. N. Engl. J. Med. NEJMoa2001282 (2020). doi:10.1056/NEJMoa2001282 Medline

10. M. L. Holshue, C. DeBolt, S. Lindquist, K. H. Lofy, J. Wiesman, H. Bruce, C. Spitters, K. Ericson, S. Wilkerson, A. Tural, G. Diaz, A. Cohn, L. Fox, A. Patel, S. I. Gerber, L. Kim, S. Tong, X. Lu, S. Lindstrom, M. A. Pallansch, W. C. Weldon, H. M. Biggs, T. M. Uyeki, S. K. Pillai, First Case of 2019 Novel Coronavirus in the United States. N. Engl. J. Med. 382, 929-936 (2020). doi:10.1056/NEJMoa2001191 Medline

11. M. Wang, R. Cao, L. Zhang, X. Yang, J. Liu, M. Xu, Z. Shi, Z. Hu, W. Zhong, G. Xiao, Remdesivir and chloroquine effectively inhibit the recently emerged novel coronavirus (2019-nCoV) in vitro. Cell Res. 30, 269-271 (2020). doi:10.1038/s41422-020-0282-0 Medline

12. J. Cohen, Can an anti-HIV combination or other existing drugs outwit the new coronavirus? Science 10.1126/science.abb0659 (27 January 2020). doi:10.1126/science.abb0659

13. Y. Chen, Q. Liu, D. Guo, Emerging coronaviruses: Genome structure, replication, and pathogenesis. J. Med. Virol. 92, 418-423 (2020). doi:10.1002/jmv.25681 Medline

14. S. Hussain, J. Pan, Y. Chen, Y. Yang, J. Xu, Y. Peng, Y. Wu, Z. Li, Y. Zhu, P. Tien, D. Guo, Identification of novel subgenomic RNAs and noncanonical transcription initiation signals of severe acute respiratory syndrome coronavirus. J. Virol. 79 , 5288-5295 (2005). doi:10.1128/JVl.79.9.5288-5295.2005 Medline

15. R. Ramajayam, K.-P. Tan, P.-H. Liang, Recent development of $3 \mathrm{C}$ and $3 \mathrm{CL}$ protease inhibitors for anti-coronavirus and anti-picornavirus drug discovery. Biochem. Soc. Trans. 39, 1371-1375 (2011). doi:10.1042/BST0391371 Medline

16. Z. Ren, L. Yan, N. Zhang, Y. Guo, C. Yang, Z. Lou, Z. Rao, The newly emerged
SARS-like coronavirus HCoV-EMC also has an "Achilles' heel": Current effective inhibitor targeting a 3C-like protease. Protein Cell 4, 248-250 (2013). doi:10.1007/s13238-013-2841-3 Medline

17. K. Anand, G. J. Palm, J. R. Mesters, S. G. Siddell, J. Ziebuhr, R. Hilgenfeld, Structure of coronavirus main proteinase reveals combination of a chymotrypsin fold with an extra $\alpha$-helical domain. EMBO J. 21, 3213-3224 (2002). doi:10.1093/emboj/cdf327 Medline

18. H. Yang, M. Yang, Y. Ding, Y. Liu, Z. Lou, Z. Zhou, L. Sun, L. Mo, S. Ye, H. Pang, G. F. Gao, K. Anand, M. Bartlam, R. Hilgenfeld, Z. Rao, The crystal structures of severe acute respiratory syndrome virus main protease and its complex with an inhibitor. Proc. Natl. Acad. Sci. U.S.A. 100, 13190-13195 (2003). doi:10.1073/pnas.1835675100 Medline

19. K. Anand, J. Ziebuhr, P. Wadhwani, J. R. Mesters, R. Hilgenfeld, Coronavirus main proteinase $\left(3 \mathrm{CL}^{\text {pro }}\right)$ structure: Basis for design of anti-SARS drugs. Science 300 , 1763-1767 (2003). doi:10.1126/science.1085658 Medline

20. F. G. Hayden, R. B. Turner, J. M. Gwaltney, K. Chi-Burris, M. Gersten, P. Hsyu, A. K. Patick, G. J. Smith 3rd, L. S. Zalman, Phase II, randomized, double-blind, placebo-controlled studies of ruprintrivir nasal spray 2-percent suspension for prevention and treatment of experimentally induced rhinovirus colds in healthy volunteers. Antimicrob. Agents Chemother. 47, 3907-3916 (2003) doi:10.1128/AAC.47.12.3907-3916.2003 Medline

21. Y. Kim, H. Liu, A. C. Galasiti Kankanamalage, S. Weerasekara, D. H. Hua, W. C. Groutas, K.-O. Chang, N. C. Pedersen, Reversal of the Progression of Fatal Coronavirus Infection in Cats by a Broad-Spectrum Coronavirus Protease Inhibitor. PLOS Pathog. 12, e1005531 (2016). doi:10.1371/journal.ppat.1005531 Medline

22. H. Yang, W. Xie, X. Xue, K. Yang, J. Ma, W. Liang, Q. Zhao, Z. Zhou, D. Pei, J. Ziebuhr, R. Hilgenfeld, K. Y. Yuen, L. Wong, G. Gao, S. Chen, Z. Chen, D. Ma, M. Bartlam, Z. Rao, Design of wide-spectrum inhibitors targeting coronavirus main proteases. PLOS Biol. 3, e324 (2005). doi:10.1371/journal.pbio.0030324 Medline

23. L. Zhang, D. Lin, Y. Kusov, Y. Nian, Q. Ma, J. Wang, A. von Brunn, P. Leyssen, K. Lanko, J. Neyts, A. de Wilde, E. J. Snijder, H. Liu, R. Hilgenfeld, $\alpha$-Ketoamides as Broad-Spectrum Inhibitors of Coronavirus and Enterovirus Replication: Structure-Based Design, Synthesis, and Activity Assessment. J. Med. Chem. acs.jmedchem.9b01828 (2020). doi:10.1021/acs.jmedchem.9b01828 Medline

24. Y. Zhai, X. Zhao, Z. Cui, M. Wang, Y. Wang, L. Li, Q. Sun, X. Yang, D. Zeng, Y. Liu, Y. Sun, Z. Lou, L. Shang, Z. Yin, Cyanohydrin as an Anchoring Group for Potent and Selective Inhibitors of Enterovirus 71 3C Protease. J. Med. Chem. 58, 9414 9420 (2015). doi:10.1021/acs.jmedchem.5b01013 Medline

25. X. Xue, H. Yang, W. Shen, Q. Zhao, J. Li, K. Yang, C. Chen, Y. Jin, M. Bartlam, Z. Rao, Production of authentic SARS-CoV Mpro with enhanced activity: Application as a novel tag-cleavage endopeptidase for protein overproduction. J. Mol. Biol. 366, 965-975 (2007). doi:10.1016/i.jmb.2006.11.073 Medline

26. W. Kabsch, Xds. Acta Crystallogr. D 66, 125-132 (2010). doi:10.1107/S0907444909047337 Medline

27. A. J. McCoy, R. W. Grosse-Kunstleve, P. D. Adams, M. D. Winn, L. C. Storoni, R. J. Read, Phaser crystallographic software. J. Appl. Crystallogr. 40, 658-674 (2007). doi:10.1107/S0021889807021206 Medline

28. P. Emsley, B. Lohkamp, W. G. Scott, K. Cowtan, Features and development of Coot. Acta Crystallogr. D 66, 486-501 (2010). doi:10.1107/S0907444910007493 Medline

29. P. D. Adams, P. V. Afonine, G. Bunkóczi, V. B. Chen, I. W. Davis, N. Echols, J. J. Headd, L.-W. Hung, G. J. Kapral, R. W. Grosse-Kunstleve, A. J. McCoy, N. W. Moriarty, R. Oeffner, R. J. Read, D. C. Richardson, J. S. Richardson, T. C. Terwilliger, P. H. Zwart, PHENIX: A comprehensive Python-based system for macromolecular structure solution. Acta Crystallogr. D 66, 213-221 (2010) doi:10.1107/S0907444909052925 Medline 


\section{ACKNOWLEDGMENTS}

We thank Prof. James Halpert and LetPub (www.letpub.com) for linguistic assistance during the preparation of this manuscript. We also thank the staff from beamlines BL17U1, BL18U1 and BL19U1 at Shanghai Synchrotron Radiation Facility (SSRF) for assistance during data collection. Funding: We are grateful to the National Natural Science Foundation of China (Nos. 21632008, 21672231, 21877118, 31970165, 91953000 and 81620108027), the Strategic Priority Research Program of the Chinese Academy of Sciences (XDA12040107 and XDA12040201) and Chinese Academy of Engineering and Ma Yun Foundation (No. 2020-CMKYGG-05) and Science and Technology Commission of Shanghai Municipality (Nos. 20431900100), and National Key R\&D Program of China (Nos. 2017YFC0840300 and 2020YFA0707500 to Z.R.), and Science and Technology Commission of Shanghai Municipality (No. 20431900200), and Department of Science and Technology of Guangxi Zhuang Autonomous Region (No. 2020AB40007), and Frontier Biotechnologies Inc. for financial support. Author contributions: H. Y. and H. L. conceived the project. Y. X., L. Z., H. Y., and H. L. designed the experiments; W. D. and J. L. designed and synthesized the compounds; $X$. J. and $H$. S tested the inhibitory activities; X. X., J. P., C. L., S. H., J. W., performed the chemical experiments and collected the data. B. Z., Y. Z., Z. J., F. L., F. B., H. W., X. C., X. L., and $X$. Y. collected the diffraction data and solved the crystal structure; Y. L. and X. C. performed the toxicity experiments. G. X., H. J., Z. R., L, Z., Y. X., H. Y. and H. L., analyzed and discussed the data. L. Z., Y. X., H. Y., and H. L., wrote the manuscript. Competing interests: The Shanghai Institute of Materia Medica has applied for PCT and Chinese patents which cover 11a, 11b and related peptidomimetic aldehyde compounds. Data and materials availability: All data are available in the main text or the supplementary materials. The PDB accession No. for the coordinates of SARSCoV-2 Mpro in complex with 11a is 6LZE, and the PDB accession No. for the coordinates of SARS-CoV-2 Mpro in complex with $11 \mathrm{~b}$ is $6 \mathrm{MOK}$. The plasmid encoding the SARS-CoV-2 Mpro will be freely available. Compounds $11 \mathrm{a}$ and $11 \mathrm{~b}$ are available from $\mathrm{H}$. L under a material transfer agreement with Shanghai Institute of Materia Medica. There is currently an international effort to join forces to design better inhibitors of SARS-CoV-2 $\mathrm{M}^{\text {pro }}$ as described in the following website: https://covid.postera.ai/covid. This work is licensed under a Creative Commons Attribution 4.0 International (CC BY 4.0) license, which permits unrestricted use, distribution, and reproduction in any medium, provided the original work is properly cited. To view a copy of this license, visit https://creativecommons.org/licenses/by/4.0/. This license does not apply to figures/photos/artwork or other content included in the article that is credited to a third party; obtain authorization from the rights holder before using such material.

\section{SUPPLEMENTARY MATERIALS}

science.sciencemag.org/cgi/content/full/science.abb4489/DC1

Materials and Methods

Scheme S1

Figs. S1 to S5

Tables S1 to S4

References (26-29)

18 March 2020; accepted 20 April 2020

Published online 22 April 2020

10.1126/science.abb4489 

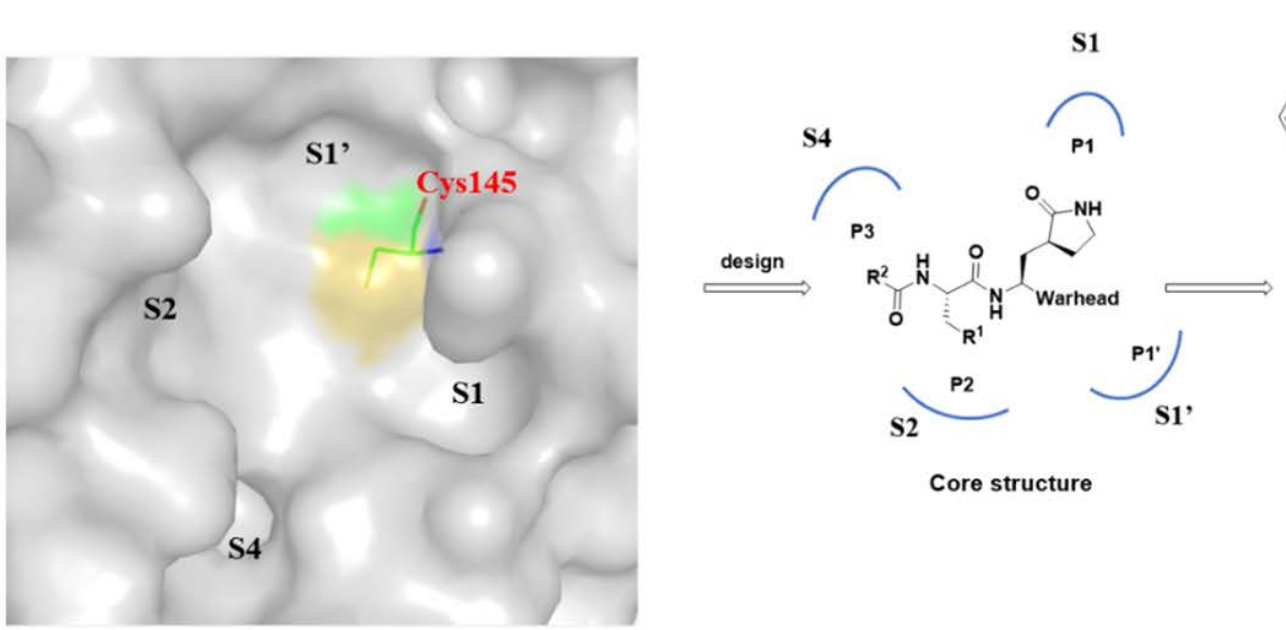

Surface representation of substrate-binding pocket of SARS-CoV $\mathbf{M}^{\text {pro }}$

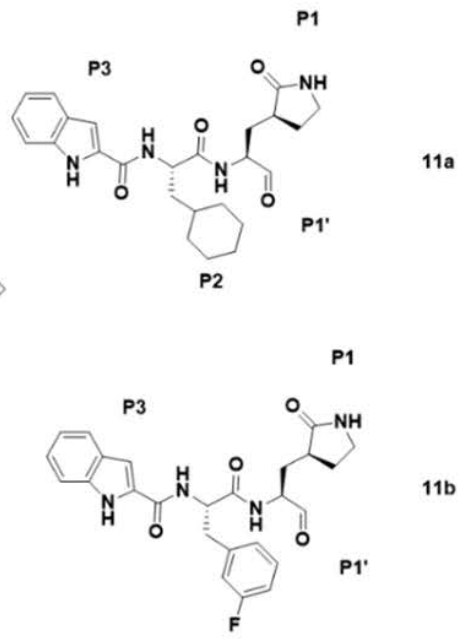

P2

Fig. 1. Design strategy of novel SARS-CoV-2 main protease inhibitors and the chemical structures of 11a and 11b.
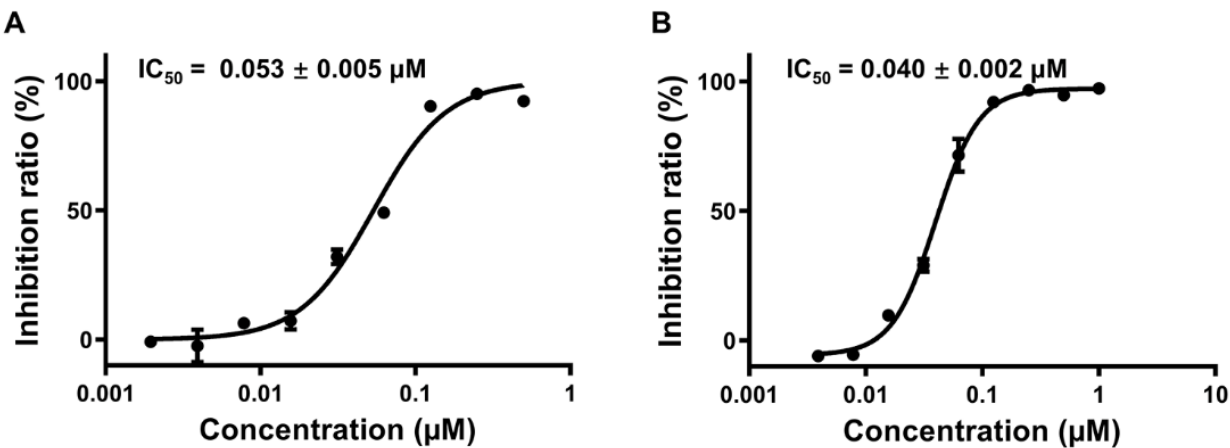

Fig. 2. Inhibitory activity profiles of compounds $11 \mathrm{a}(\mathrm{A})$ and $11 \mathrm{~b}(\mathrm{~B})$ against SARS-CoV-2 $\mathrm{M}^{\text {pro }}$. 

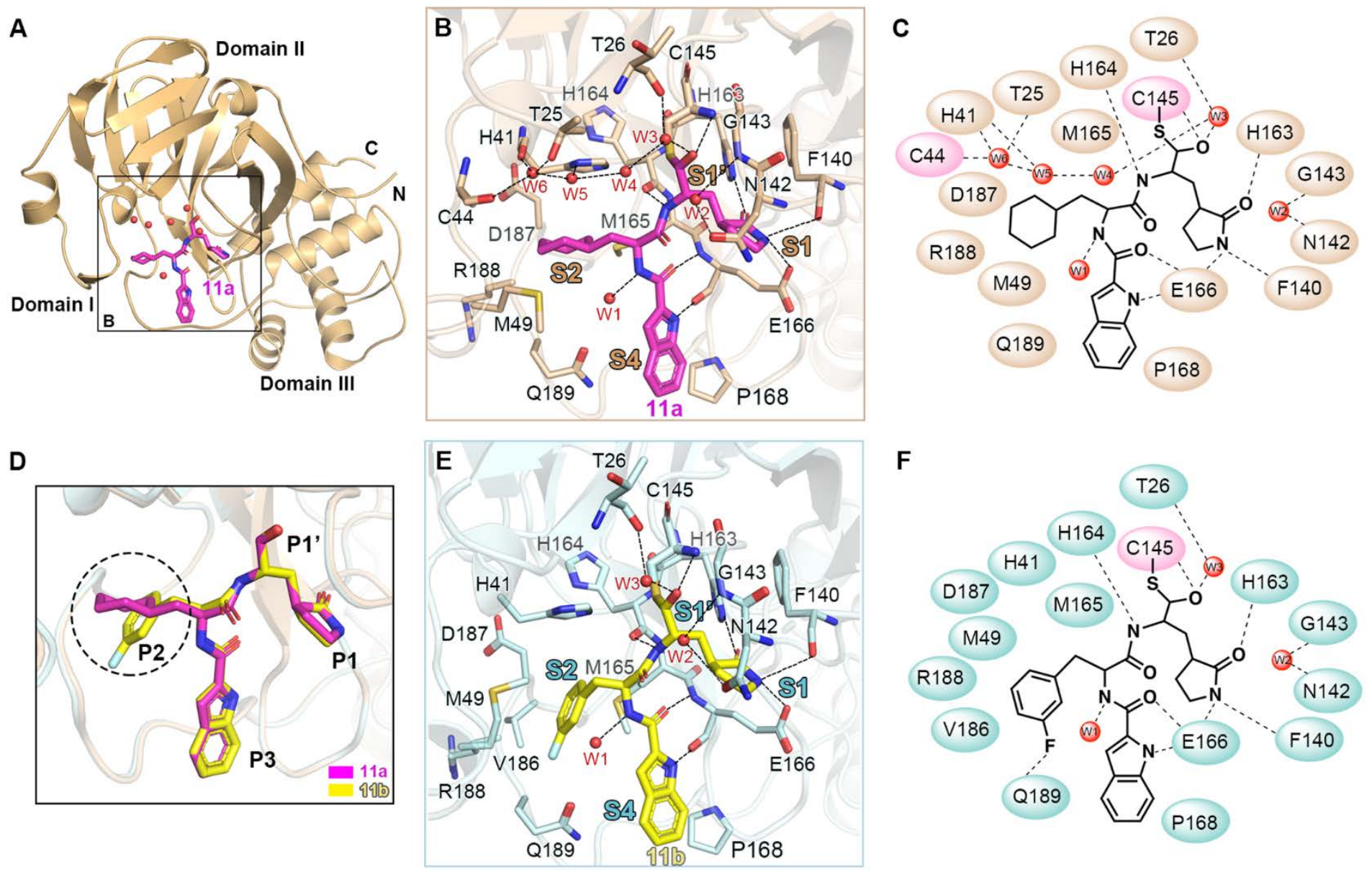

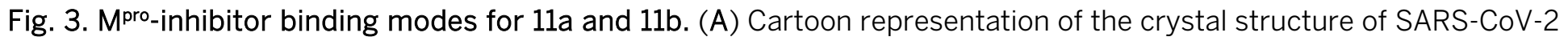
$\mathrm{M}^{\text {pro }}$ in complex with 11a. The compound 11a is shown as magenta sticks; water molecules shown as red spheres. (B) Close-up view of the 11a binding pocket. Four subsites, S1', S1, S2 and S4, are labeled. The residues involved in inhibitor binding are shown as wheat sticks. 11a and water molecules are shown as magenta sticks and red spheres, respectively. Hydrogen bonds are indicated as dashed lines. (C) Schematic diagram of SARS-CoV-2 Mpro-11a interactions shown in (B). (D) Comparison of the binding modes between 11a and 11b for SARS-CoV-2 Mpro. The major differences between 11a and $11 \mathrm{~b}$ are marked with dashed circles. The compounds of $11 \mathrm{a}$ and $11 \mathrm{~b}$ are shown as magenta and yellow sticks, respectively. (E) Close-up view of the $11 \mathrm{~b}$ binding pocket. Hydrogen bonds are indicated as dashed lines. (F) Schematic diagram of SARS-CoV-2 Mro-11b interactions shown in (E). 

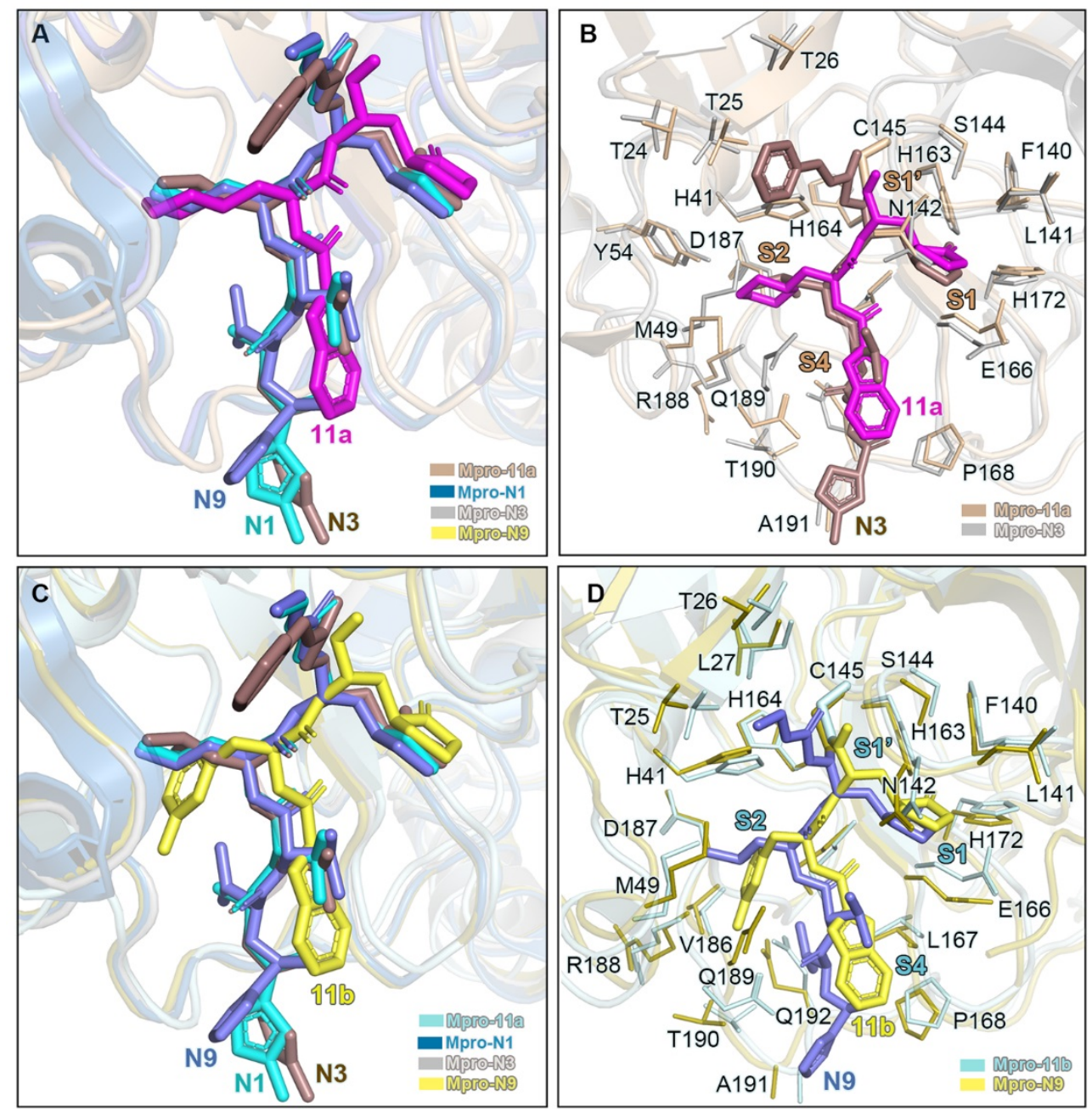

Fig. 4. Comparison of the inhibitor binding modes in SARS-CoV and SARS-CoV-2 $\mathrm{M}^{\text {pros. }}$. (A) Comparison of binding modes of 11 a in SARS-CoV-2 $\mathrm{M}^{\text {pro }}$ with those of N1, N3 and N9 in SARS-CoV M ${ }^{\text {pro. }}$. SARS-CoV-2 Mpro-11a (wheat, PDB code: 6LZE), SARS-CoV Mpro-N1 (sky blue, PDB code:1WOF), SARS-CoV Mpro-N3 (gray, PDB code: 2AMQ) and SARS-CoV Mpro-N9 (olive, PDB code: 2AMD).11a, N1, N3 and N9 are shown in magenta, cyan, dirty violet and salt, respectively. (B) Comparison of the 11a and N3 binding pockets. Residues in Mpro-11a structure and

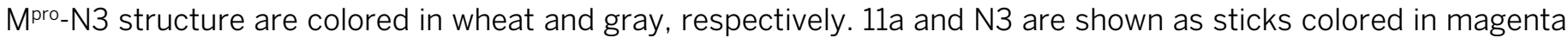
and dirty violet, respectively. (C) Comparison of binding modes of 11b in SARS-CoV-2 Mro with those of N1, N3 and N9 in SARS-CoV Mpro. SARS-CoV-2 Mpro-11b (pale cyan, PDB code: 6MOK). 11b, N1, N3 and N9 are shown in yellow, cyan, dirty violet and salt, respectively. (D) Comparison of the $11 \mathrm{~b}$ and $\mathrm{N} 9$ binding pockets. Residues in $\mathrm{M}^{\text {pro- }}-11 \mathrm{~b}$

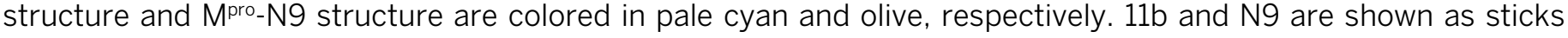
colored in yellow and salt, respectively. 
A
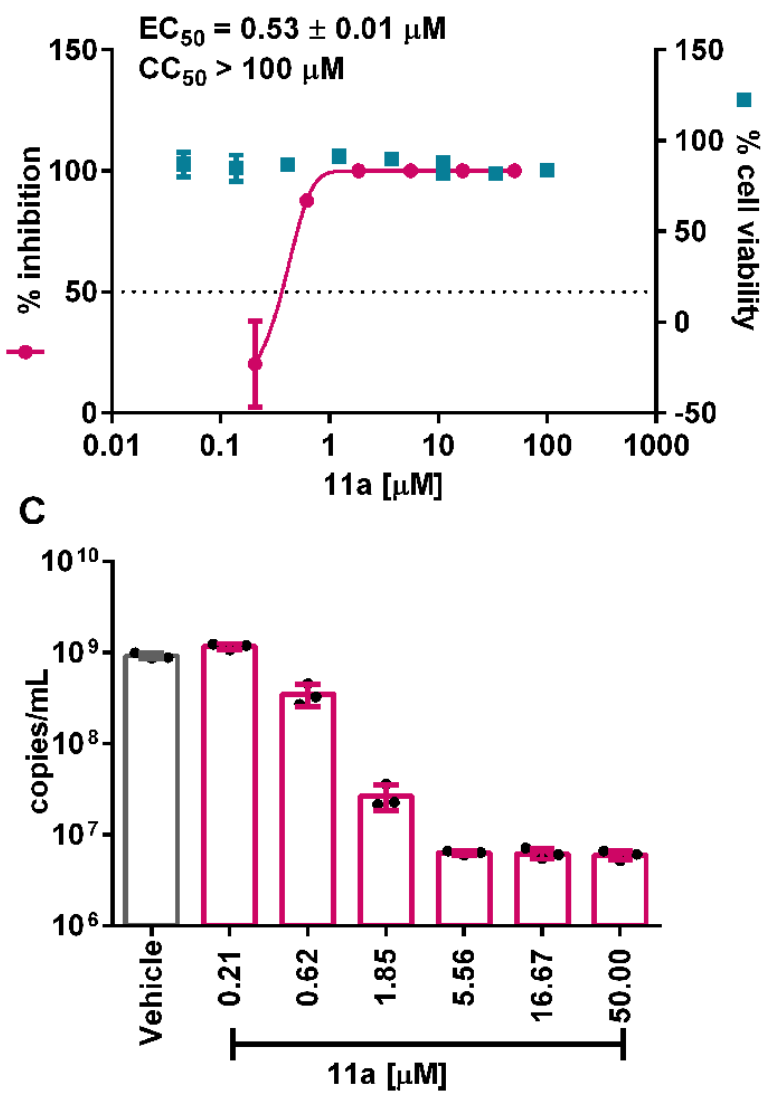

B
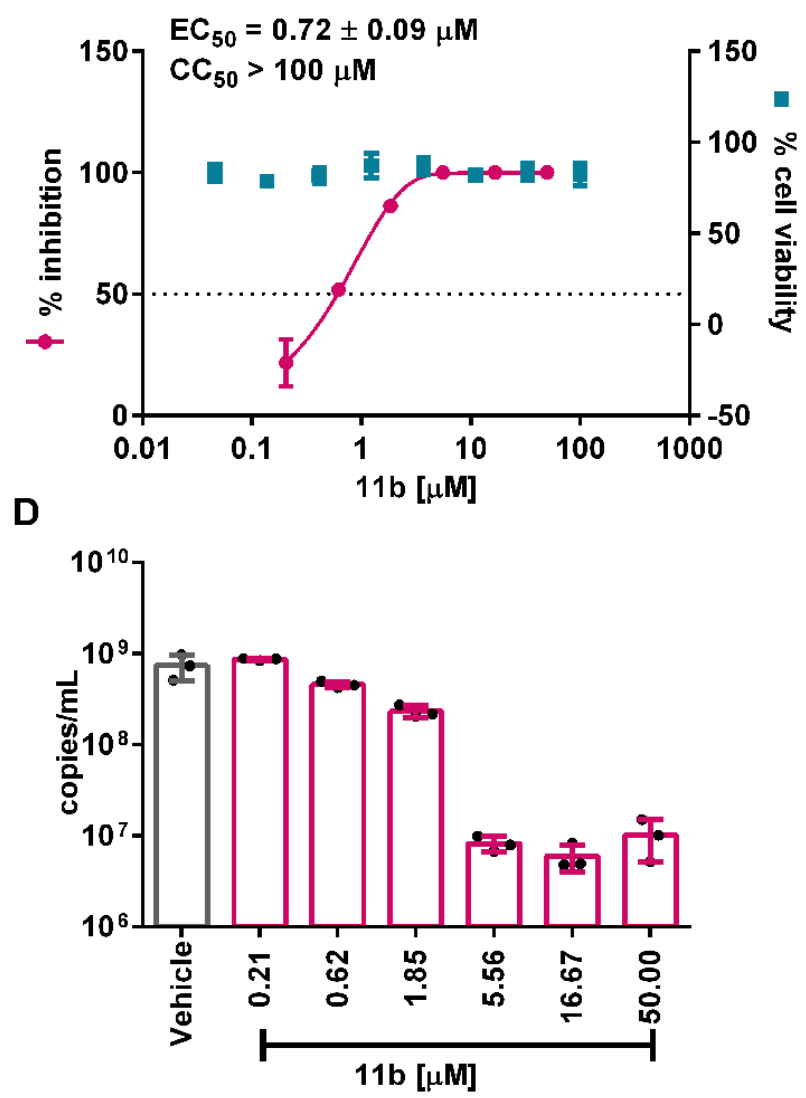

Fig. 5. In vitro inhibition of viral main protease inhibitors against SARS-CoV-2. (A and B) Vero E6 cells were treated with a series concentration of indicated compounds $11 \mathrm{a}$ and $11 \mathrm{~b}$ and infected with SARS-CoV-2 at an MOI of 0.05 . At 24 hours post infection, viral yield in the cell supernatant was quantified by plaque assay. The cytotoxicity of these compounds in Vero E6 cells was also determined by using CCK8 assays. The left and right Y-axis of the graphs represent mean \% inhibition of virus yield and mean \% cytotoxicity of the drugs, respectively. (C and $\mathrm{D})$ Viral RNA copy numbers in the cell supernatants were quantified by qRT-PCR. Data are mean $\pm S D, n=3$ biological replicates. 